\title{
What Do Interval Cycles Have To Do With Tonal Harmony?
}

\author{
IAN QUINN \\ Yale University
}

\begin{abstract}
Woolhouse's (2010) central empirical finding is a relatively high correlation between his interval-cycle proximity (ICP) model and the theorist Walter Piston's (1979) Table of Usual Root Progressions. The fit between these two models can be understood in terms of a classification of chord progressions by root interval class (second, third, fifth) and directionality (strong, weak). The ICP model does not perform as well on data on chord progressions in Tymoczko's (forthcoming) corpora of music by Bach and Mozart. The alternative MHP model (Quinn, 2010) does not fit the Piston data as well as the ICP model, but it fits the corpus data better than the ICP model.
\end{abstract}

Submitted 2010 July 6; accepted 2010 July 25.

KEYWORDS: tonal attraction, chord progressions, tonal harmony, corpus analysis

WALTER Piston's 1941 harmony book — still in print in a 1987 revision by Mark DeVoto — has largely been supplanted by newer textbooks, but two of its coinages are still in wide circulation. First is the term common practice, a term Piston uses to conjure a unified vocabulary and syntax for music of the eighteenth and nineteenth centuries, factoring out differences of style and genre. His stated aim seems to have been to observe and describe the music of the past for the benefit of the composers of the present, and not to explain how the past came to be, or to prescribe what the future ought to sound like.[1] Piston is clearly not a historicist; the raison d'être of his idealized, homogenized concept of a common practice seems to be that it might serve as a matrix for the music of the present. He does, however, profess to be an empiricist: "Rules," he avers in the book's introduction, "are announced as observations reported, without attempt at their justification on aesthetic grounds or as laws of nature." Piston's summary presentation of these rules, the "Table of Usual Root Progressions," is the other lasting feature of the book. It takes the form of an approximate first-order transition table for chord roots: rules take the form "IV is followed by V, sometimes I or II, less often III or VI."

Piston's table has continued to attract the interest of empirically minded music researchers as a touchstone, or ground truth, for models of tonal harmony (Meyer, 1956; Schmuckler, 1989; Bharucha \& Olney, 1989; Povel, 2002; Ockelford 2006). Matthew Woolhouse's article (2010) is no exception. Woolhouse has developed a general theory of tonal attraction (Woolhouse, 2007, 2009; Woolhouse and Cross, 2010), and the central empirical finding of the article considered here is a significant rank-order correlation between the attraction values for the 49 possible progressions between diatonic triads and a set of ratings for the typicality of those progressions derived from Piston's Table of Usual Root Progressions. Encouraged by these results, Woolhouse offers some speculations about how Piston's "common practice" emerged from sixteenth-century modal practice.

\section{MEEÙS'S CLASSIFICATION OF CHORD PROGRESSIONS}

Table 1, adapted from Woolhouse's Table 4, compares Piston's estimates of chord-transition likelihood (unshaded numbers) with the chord-attraction values output by Woolhouse's model (shaded numbers). The Spearman rank-order correlation between these two models is $r_{\mathrm{s}}=0.68$, a number Woolhouse interprets as indicating "broad agreement" between them. DeVoto's 1987 revision of Piston, which Woolhouse does not seem to have consulted, includes some adjustments to the Table of Usual Root Progressions. These adjustments are indicated in parentheses in Table 1; when they are taken into account the correlation with Woolhouse's model reaches an even more impressive $r_{\mathrm{s}}=0.81$. 


\begin{tabular}{c|cc|cc|cc|cc|cc|cc|cc} 
& \multicolumn{2}{|c|}{ I } & \multicolumn{2}{|c|}{ ii } & \multicolumn{2}{|c|}{ iii } & \multicolumn{2}{c|}{ IV } & \multicolumn{2}{|c|}{ V } & \multicolumn{2}{|c}{ vi } & vii \\
\hline I & 0.30 & 1 & $\mathbf{0 . 6 0}$ & $\mathbf{2}$ & 0.35 & 2 & $\mathbf{0 . 8 0}$ & $\mathbf{4}$ & 0.73 & 4 & $\mathbf{0 . 4 2}$ & $\mathbf{3}$ & 0.39 & 1 \\
ii & 0.59 & 2 & 0.32 & 1 & $\mathbf{0 . 6 5}$ & $\mathbf{2}$ & 0.35 & 3 & $\mathbf{0 . 7 2}$ & $\mathbf{4}$ & 0.7 & 3 & $\mathbf{0 . 1 4}$ & $\mathbf{1}$ \\
iii & $\mathbf{0 . 4 5}$ & $\mathbf{2}$ & 0.60 & 2 & 0.32 & 1 & $\mathbf{0 . 7 5}$ & $\mathbf{3}$ & 0.35 & 2 & $\mathbf{0 . 7 4}$ & $\mathbf{4}$ & 0.34 & 1 \\
IV & 0.73 & 3 & $\mathbf{0 . 4 2}$ & $\mathbf{3}$ & 0.79 & 2 & 0.30 & 1 & $\mathbf{0 . 5 5}$ & $\mathbf{4}$ & 0.35 & 2 & $\mathbf{0 . 1 8}$ & $\mathbf{1}$ \\
V & $\mathbf{0 . 8 0}$ & $\mathbf{4}$ & 0.68 & 2 & $\mathbf{0 . 4 2}$ & $\mathbf{2}$ & 0.48 & 3 & 0.30 & 1 & $\mathbf{0 . 6 0}$ & $\mathbf{3}$ & 0.23 & 1 \\
vi & 0.35 & 2 & $\mathbf{0 . 7 4}$ & $\mathbf{4}$ & 0.7 & 3 & $\mathbf{0 . 4 5}$ & $\mathbf{3}$ & 0.59 & 4 & 0.32 & 1 & $\mathbf{0 . 3 3}$ & $\mathbf{1}$ \\
vii & $\mathbf{1 . 2 1}$ & $\mathbf{4}$ & 0.41 & $1(2)$ & $\mathbf{1 . 1 1}$ & $\mathbf{3 ( 4 )}$ & 0.44 & $1(2)$ & $\mathbf{0 . 7 6}$ & $\mathbf{1 ( 2 )}$ & 0.95 & $1(3)$ & 0.21 & 1
\end{tabular}

Table 1. Woolhouse's attraction values (shaded) and Piston's chord-transition estimates (unshaded) for progressions from one diatonic chord (rows) to another (columns). Boldface figures indicate strong chord progressions; italicized figures indicate weak chord progressions.
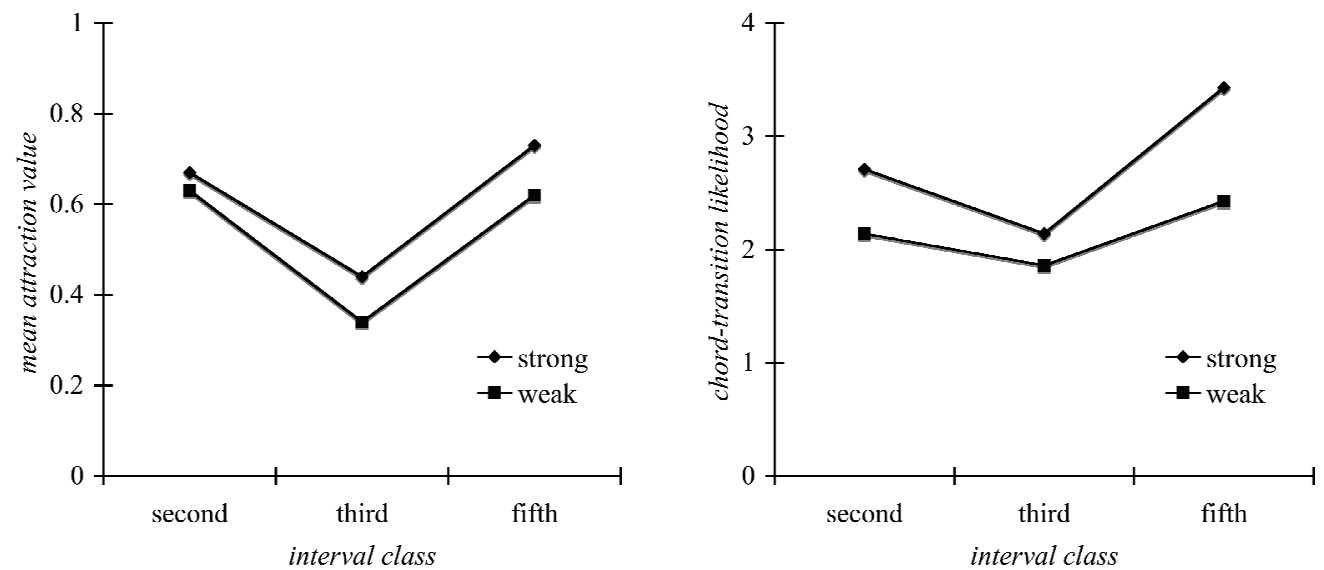

Figure 1. Analysis of data in Table 1, showing main effects of root interval class and directionality on Woolhouse's attraction values (left) and Piston's chord-transition estimates (right).

Let us take a closer look at this "broad agreement." Some cells in Table 1 contain boldface numbers, and others contain italicized numbers. The particularly observant reader will have noticed that the boldface numbers are, on the whole, greater than the italicized numbers. The typographic asymmetry reflects a theoretical asymmetry among chord progressions that was suggested by Schoenberg (1954), worked out by Meeùs (2000), and further contextualized by Tymoczko (2003). What Meeùs observed, at Schoenberg's prompting, was that chord progressions involving a descending fifth (as iii-vi), descending third (as IV-ii), or ascending second (as IV-V) are stronger and more common than chord progressions by ascending fifth (as vi-iii), ascending third (as ii-IV), or descending second (as V-IV). Meeùs refers to chord progressions of the former category (strong and frequent) as dominant, and to those of the latter category (weak and infrequent) as subdominant. I shall use the less musictheoretically freighted terms strong and weak instead. The opposition between strong and weak progressions is what gives tonal harmony a directional character: the retrograde of every strong progression is a weak progression, and vice versa. We can therefore characterize any diatonic chord progression in terms of two independent factors: the interval class by which the root moves (second, third, or fifth) and the directionality of that motion (strong or weak).

Boldface numbers in Table 1 correspond to strong progressions, and italicized numbers to weak progressions. On average, Piston predicts a chord will be more likely to be followed by a chord forming a strong progression than one forming a weak progression: strong progressions have a mean likelihood of 2.76, compared to 2.14 for weak progressions (see Figure 1). Likewise, Woolhouse's model asserts a stronger attraction from the first chord to the second in a strong progression (mean attraction of 0.62 ) than in a weak progression (0.53). Not only do the Piston and Woolhouse models agree on this effect of directionality, but they also agree on the effect of root interval class. For one thing, among strong progressions, descending fifths are strongest on average (3.43 for Piston, 0.73 for Woolhouse), followed by ascending seconds (2.71 and 0.67 ), with descending thirds coming in last (2.14 and 0.44$)$. When it comes to weak progressions, the hierarchy of intervals is basically preserved: 
the strongest of the weak progressions are the ascending fifths $(2.43$ and 0.62$)$ and the descending seconds (2.14 and 0.63$)$, followed by the ascending thirds (1.86 and 0.34 ).

\section{WHY WOOLHOUSE'S CHORD MODEL SUCCEEDS}

Piston's model is a black box, but Woolhouse's is transparent, and we are thereby able to see clearly why it manages to produce attraction values that reflect the effects of both progression directionality (strong versus weak) and root interval class (fifth versus second versus third). Before turning to this explanation, some details of Woolhouse's model must be reviewed. The model is based on what Woolhouse calls the Interval Cycle Proximity (ICP) Hypothesis, which he characterizes as follows:

Tonal attraction is proportional to the sum of the interval cycles formed between sequential pairs of pitches and/or chords. High interval cycles lead to strong tonal attraction; low interval cycles lead to weak tonal attraction.

For the discussion that follows, it will be convenient to separate this into two more or less independent components: a pitch-class model and a chord model. The pitch-class model proposes that the tonal attraction between two notes separated by an interval of $i$ semitones is directly proportional to the smallest positive integer $c$ such that $c i=0(\bmod 12)$. This number $c$ is what Woolhouse calls the interval-class proximity (ICP) of the pitch-classes in question. The chord model scales the pitch-class model up to collections of pitches, hypothesizing that the tonal attraction between two pitch collections $X=\left\{x_{0}, x_{1}, \ldots, x_{m}\right\}$ and $Y=\left\{y_{0}, y_{1}, \ldots, y_{n}\right\}$ is simply a weighted average of the attractions between individual pairs of notes $\left(x_{i}, y_{j}\right)$. Woolhouse has some specific ideas about what the weights should be, suggesting that certain intervals be given additional weight in cases where one or more of the chords in question has an identifiable root and/or is dissonant. For example, given a progression between two consonant triads, the Root Salience component of his model multiplies the attraction values for intervals involving the first chord's root by $\beta>1$, and likewise multiplies the attraction values for intervals involving the second chord's root by $\gamma>\beta$.

The ICP-based pitch-class model has two curious side effects. The first is that if the intervals $i$ and $j$ and inversions of each other, that is if $i=-j(\bmod 12)$, then they will have the same attraction value. Since the effect of running an interval backwards is to invert it[2], Woolhouse's commitment to an ICP-based pitch-class model smuggles in an assumption that tonal attraction is invariant under both pitch-class inversion and retrogradation. His scheme for differentially weighting intervals in the chord model is partly designed to overcome this assumption.

The second side effect of the pitch-class model is that the seven chromatic interval classes can be collapsed onto the four diatonic interval classes while (mostly) maintaining a hierarchy of tonal attraction. That is, both varieties of diatonic second ( 1 and 2 semitones) have higher ICP values (12 and 6 , respectively) than both varieties of diatonic third ( 3 and 4 semitones, with ICP values of 4 and 3 , respectively), and all of these have higher ICP values than the unison ( 0 semitones, ICP $=1)$. The only exception to this is the tritone $(6$ semitones, ICP $=2)$, which has a markedly lower ICP than its diatonic cousin, the perfect fourth ( 5 semitones, ICP $=12$ ).

We are now in a position to understand why Woolhouse's model shows an effect of root interval class. Of the nine intervals involved in a progression from one consonant three-note chord to another, the one that gets the most weight in Woolhouse's chord model is the interval between the roots: the attraction value between the roots is multiplied both by $\beta$ and by $\gamma$. With Woolhouse's values of $\beta=4$ and $\gamma=8$, the attraction value of this interval alone will account for slightly more than half of the weighted average that determines the attraction value of the progression as a whole. For this reason, the hierarchy of tonal attraction among diatonic intervals is in large part maintained when chords are built on those intervals. Root motions by fifth (which are always perfect in the case of diatonic consonant triads) will always have an ICP of 12; root motions by second will have an ICP of either 12 or 6; and root motions by third will always have an ICP of 4 or 3. And so, as we have seen, chord progressions by fifth tend to have an attraction value that is the same as or higher than chord progressions by second, and chord progressions by third tend to have relatively low attraction values. 


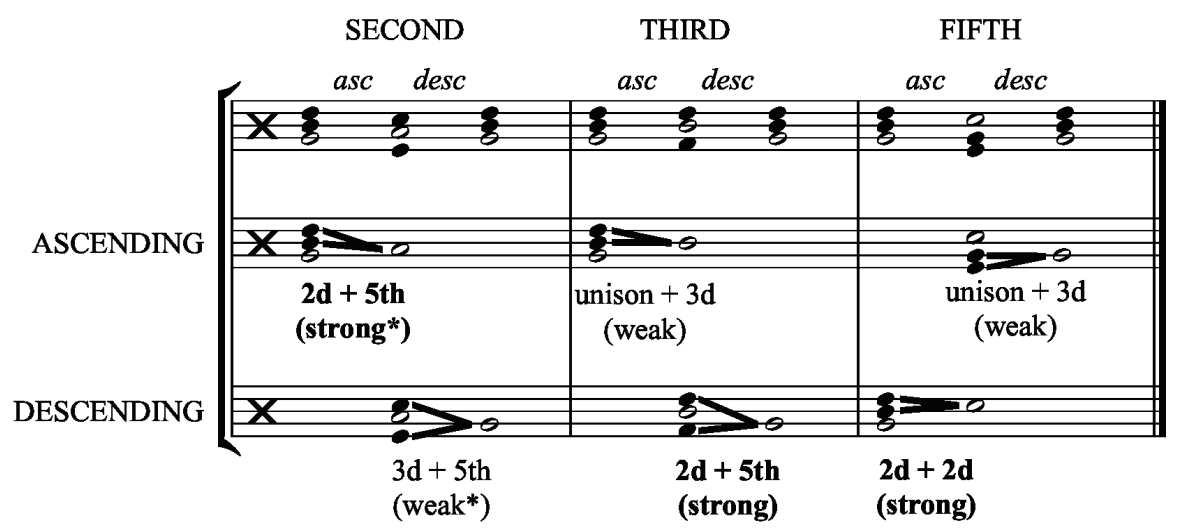

Figure 2. Interval classes between the non-root members of the first chord and the root of the second chord in the six types of chord progression, by root interval class (left to right). An ascending fifth is the same as a descending fourth, and vice versa.

The effect of directionality (strong versus weak) requires a bit more explanation. The difference between the attraction values of a progression and its retrograde depends on two factors: (a) the values of $\beta$ and $\gamma$ and (b) the attraction values of the intervals between the root of one chord and the non-root tones of the other. If we label the two chords $x$ (comprising root $x_{\mathrm{R}}$, third $x_{3}$, and fifth $x_{5}$ ) and $y$ (comprising $y_{\mathrm{R}}, y_{3}$, and $y_{5}$ ), and refer to the attraction value of the interval from $p$ to $q$ as $a(p, q)$, then the attraction value of the progression from $x$ to $y$ is

$$
A(x, y)=\frac{\beta \gamma a\left(x_{\mathrm{R}}, y_{\mathrm{R}}\right)+\gamma a\left(x_{3}, y_{\mathrm{R}}\right)+\gamma a\left(x_{5}, y_{\mathrm{R}}\right)+\beta a\left(x_{\mathrm{R}}, y_{3}\right)+\beta a\left(x_{\mathrm{R}}, y_{5}\right)+a\left(x_{3}, y_{3}\right)+a\left(x_{3}, y_{5}\right)+a\left(x_{5}, y_{3}\right)+a\left(x_{5}, y_{5}\right)}{12(\beta \gamma+2 \beta+2 \gamma+4)}
$$

Because Woolhouse's ICP model gives an interval and its inverse the same attraction value, the difference in attraction values between a chord progression and its retrograde can be expressed as

$$
\begin{aligned}
A(x, y)-A(y, x) & =\frac{\gamma a\left(x_{3}, y_{\mathrm{R}}\right)+\gamma a\left(x_{5}, y_{\mathrm{R}}\right)+\beta a\left(x_{\mathrm{R}}, y_{3}\right)+\beta a\left(x_{\mathrm{R}}, y_{5}\right)-\left[\gamma a\left(y_{3}, x_{\mathrm{R}}\right)+\gamma a\left(y_{5}, x_{\mathrm{R}}\right)+\beta a\left(y_{\mathrm{R}}, x_{3}\right)+\beta a\left(y_{\mathrm{R}}, x_{5}\right)\right]}{12(\beta \gamma+2 \beta+2 \gamma+4)} \\
& =k[W(x, y)-W(y, x)],
\end{aligned}
$$

where $\mathrm{W}(\mathrm{x}, \mathrm{y})$ is equal to the sum of the attraction values from the third and fifth of $\mathrm{x}$ to the root of $\mathrm{y}$ :

$$
W(x, y)=a\left(x_{3}, y_{\mathrm{R}}\right)+a\left(x_{5}, y_{\mathrm{R}}\right),
$$

and where $\mathrm{k}$ is a constant determined by $\beta$ and $\gamma$ :

$$
k=\frac{(\gamma-\beta)}{12(\beta \gamma+2 \beta+2 \gamma+4)}
$$




\begin{tabular}{c|ccccccc} 
& I & ii & iii & IV & V & vi & vii \\
\hline I & $*$ & $\mathbf{. 1 7}$ & .00 & $\mathbf{. 3 0}$ & .43 & $\mathbf{. 0 7}$ & .02 \\
ii & .00 & $*$ & $\mathbf{. 0 0}$ & .00 & $\mathbf{. 7 0}$ & .00 & $\mathbf{. 3 0}$ \\
iii & .00 & .00 & $*$ &. $\mathbf{6 7}$ & .00 & $\mathbf{. 3 3}$ & .00 \\
IV & .29 & $\mathbf{. 0 8}$ & .03 & $*$ & $\mathbf{. 3 7}$ & .01 & $\mathbf{. 2 2}$ \\
V & $\mathbf{. 8 5}$ & .00 & $\mathbf{. 0 0}$ & .07 & $*$ & $\mathbf{. 0 8}$ & .00 \\
vi & .15 & $\mathbf{. 3 1}$ & .04 & $\mathbf{. 2 7}$ & .15 & $*$ & $\mathbf{. 0 8}$ \\
vii &. $\mathbf{9 7}$ & .00 & $\mathbf{. 0 0}$ & .03 & $\mathbf{. 0 0}$ & .00 & $*$
\end{tabular}

\begin{tabular}{c|ccccccc} 
& I & ii & iii & IV & V & vi & vii \\
\hline I & $*$ & $\mathbf{. 1 2}$ & .00 & $\mathbf{. 1 4}$ & .63 & $\mathbf{. 0 6}$ & .05 \\
ii & .01 & $*$ & $\mathbf{. 0 0}$ & .01 & $\mathbf{. 8 9}$ & .01 & $\mathbf{. 0 8}$ \\
iii & .57 & .00 & $*$ & .00 & .00 & $\mathbf{. 4 3}$ & .00 \\
IV & .34 &. $\mathbf{1 7}$ & .00 & $*$ &. $\mathbf{4 3}$ & .00 & $\mathbf{. 0 6}$ \\
V & .93 & .00 & $\mathbf{. 0 0}$ & .01 & $*$ & $\mathbf{. 0 4}$ & .01 \\
vi & .09 & $\mathbf{. 4 6}$ & .00 & $\mathbf{. 1 6}$ & .24 & $*$ & $\mathbf{. 0 5}$ \\
vii & $\mathbf{. 7 8}$ & .00 & $\mathbf{. 0 0}$ & .01 & $\mathbf{. 2 1}$ & .00 & $*$
\end{tabular}

Table 2. First-order transition probabilities for chords in a corpus of Bach chorales (left) and Mozart piano sonatas (right). From Tymoczko (forthcoming).
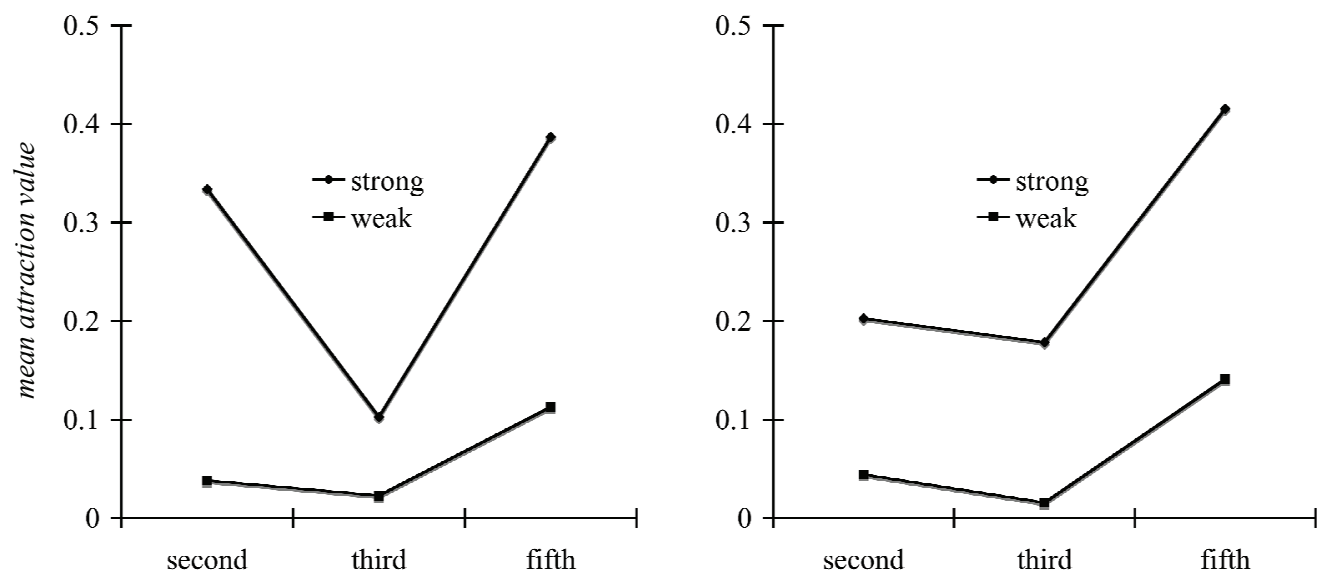

Figure 3. Average attraction of each progression type as estimated from the transition probabilities in the Bach (left) and Mozart (right) corpora shown in Table 2.

So the requirement that $\gamma$ be greater than $\beta$ ensures that the difference between $\mathrm{A}(\mathrm{x}, \mathrm{y})$ and $\mathrm{A}(\mathrm{y}, \mathrm{x})$ will necessarily be directly proportional to the difference between $\mathrm{W}(\mathrm{x}, \mathrm{y})$ and $\mathrm{W}(\mathrm{y}, \mathrm{x})$. And as it turns out, $\mathrm{W}(\mathrm{x}, \mathrm{y})$ is greater than $\mathrm{W}(\mathrm{y}, \mathrm{x})$ if and only if $\mathrm{x} \rightarrow \mathrm{y}$ is a strong progression, with just one exception. Figure 2 shows why this is so. For all six types of chord progressions (ascending and descending root motions of a second, third, and fifth), the figure highlights the intervals involved in calculating W(x,y), i.e. the intervals from the third and fifth of $\mathrm{x}$ to the root of $\mathrm{y}$. For every strong progression these intervals are either both seconds, or one is a second and the other is a fourth/fifth. These are the intervals associated with the highest ICP values - perfect fourths/fifths and minor seconds have an ICP of 12, and major seconds have an ICP of 6 . Every weak progression, on the other hand, has a third (ICP $=3$ or 4$)$ as one of these intervals, and most have a unison $(\mathrm{ICP}=1)$ as the other.

\section{TESTING WOOLHOUSE’S MODEL ON CORPUS DATA}

Thus far we have been concerned with explaining the correlation between the attraction values produced by Woolhouse's model and Piston's Table of Usual Root Progressions, the central empirical finding of Woolhouse's article. It must be said, however, that Piston's textbook has fallen into disuse despite DeVoto's efforts at revision, and the Table of Usual Root Progressions no longer has currency as a pedagogical model for teachers of music theory. Piston's table reflects his intuitions about an idealized and ideologically motivated "common practice." It does not reflect the facts of any musical corpus. The opposite approach is taken by Tymoczko (forthcoming), who, inspired by McHose (1937) and Budge (1943), analyzes chord frequencies in two corpora - selected Bach chorales and selected Mozart piano-sonata movements - as first-order Markov processes. ${ }^{3}$ Tymoczko's transition tables for chords are shown in Table 2, with strong and weak progressions indicated typographically as in Table 1. We can treat these probabilities as an empirical index of the attraction from one chord to another. Figure 3 shows that there are effects of both root interval class and directionality on these empirically 
derived attraction values. Indeed, both corpora show both effects to a much stronger degree than either Woolhouse's model or the Piston-derived typicality ratings. The effect of directionality (strong versus weak) is especially exaggerated in the corpus data. The corpora also show a much sharper distinction between progressions by third (low attraction) and by fifth (high attraction) than the two theoretical models.

It would seem that the Piston-derived typicality ratings understate the differences between progressions that happen and those that do not happen. To point out just a few cases, Piston rates iiiIV, ii-vi, and vi-iii as belonging to the second most typical category of progressions, and Woolhouse's model produces high attraction values for them, yet all are quite rare in both the Bach and Mozart corpora. The question now arises whether the ICP model is as highly correlated with Tymoczko's transition tables as it is with Piston's table. Before the ICP model's attraction values can be directly compared to probabilities, they must be normalized row by row. This is accomplished by dividing the attraction value of chord $x$ to chord $y$ by the total of the attraction values from chord $x$ to each of the seven chords. The normalized attraction values can then be directly compared to Tymoczko's chordtransition data using Pearson's product-moment correlation method instead of the Spearman rank-order correlation method. As it turns out, the ICP model is a better fit with Piston's table than with the corpus data (Bach corpus: $r=0.51$; Mozart corpus: $r=0.49$ ). Like Piston, Woolhouse's model underestimates the effect of root-motion directionality and overstates the attractive power in chord progressions by third.[4]

\begin{tabular}{c|cccccccccccc} 
semitones & 0 & +1 & +2 & +3 & +4 & +5 & +6 & +7 & +8 & +9 & +1 & +1 \\
& & & & & & & & & & & 0 & 1 \\
\hline MHP & 0 & 12 & 6 & 0 & 0 & 12 & 0 & 6 & 0 & 0 & 3 & 6 \\
\hline ICP & 1 & 12 & 6 & 4 & 3 & 12 & 2 & 12 & 3 & 4 & 6 & 12
\end{tabular}

Table 3. Comparison of attraction values for intervals in Woolhouse's ICP model and hypothetical MHP (melodic-harmonic proximity) model.

\section{WHY WOOLHOUSE'S PITCH-CLASS MODEL DOES NOT SUCCEED}

Elsewhere (Quinn, 2010) I have proposed a modification to Woolhouse's pitch-class model that preserves his chord model while swapping out the underlying pitch-class model. Instead of intervalclass proximity, this alternative model is based on two ideas: (a) ascending and descending steps and fifths alone are the basis of attraction, and (b) an interval does not necessarily have the same attractive power as its inversion. This pitch-class model, which I called melodic-harmonic proximity (MHP), is presented alongside Woolhouse's ICP pitch-class model in Table 3. The MHP-based method of determining attraction values for chord progressions is identical to Woolhouse's procedure in all other respects, i.e. those pertaining to the chord model.

The goal of comparing the performance of the ICP pitch-class model and the MHP pitch-class model in Woolhouse's attraction-value algorithm is to test whether interval-class proximity has as much explanatory value as Woolhouse claims for it. The two models are based on two unrelated claims about how tonal attraction works, yet they make reasonably close predictions about attractions between single pitch-classes. This correlation is coincidental, an effect of the law of small numbers. Suppose the MHP-based model performs better than the ICP-based model. This would give us reason to accept the MHP hypothesis and to reject the ICP hypothesis, accruing the success of the ICP-based model to the correlation between the two pitch-class models. In fact, the MHP-based chord model has proved to explain Woolhouse's other data better than his own ICP-based chord model (Quinn, 2010). If it turns out that it also explains the chord-progression data better than the ICP model, further doubt will be cast on the ICP Hypothesis. 


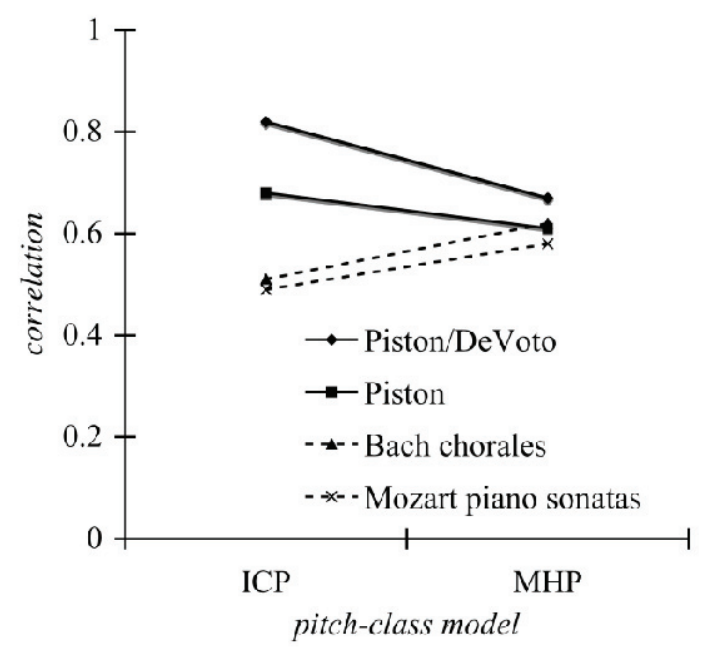

Figure 4. Comparison of the performance of Woolhouse's model on the Piston and corpus data with Woolhouse's original ICP-based pitch-class model and the MHP-based pitch-class model.

Figure 4 shows how the MHP model performs in comparison to the ICP model on the data we have been working with in this paper. With respect to both the Piston table used by Woolhouse and the table derived from DeVoto's revision of Piston, the MHP model does worse than the ICP model (Piston: $r_{\mathrm{s}}=0.61$; Piston/Devoto: $r_{\mathrm{s}}=0.68$ ). Thus as an explanatory model for Piston's Table of Usual Root Progressions, ICP is superior. But if we leave Piston aside and turn to the more important question of the models' fit with the corpus data, we find that the MHP-based version of Woolhouse's model fits the data substantially better than does Woolhouse's original ICP-based model (Bach corpus: $r=0.62$; Mozart corpus: $r=0.58$ ), as Figure 4 shows. And this finding, on its own, constitutes prima facie evidence that the MHP Hypothesis is more likely to be correct than the ICP Hypothesis.

What advantage does the MHP pitch-class model have over the ICP model? An important difference between the two is that many intervals have an MHP value of 0 , and a positive ICP value. The intervals having this property include the major and minor thirds and sixths, plus the unison/octave - precisely the intervals given extra weight by Woolhouse's chord model when faced with a weak progression (recall Figure 2). As a result, the attraction values for chord progressions in the ICP model show a floor effect. When converted to transition probabilities in the manner described above, ICP attraction values for consonant diatonic chord progressions get no lower than 0.096 (for I-iii), and the lowest attraction value for any nontrivial chord progression is .042 (for ii-vii ${ }^{\circ}$ ). The corresponding minima for the MHP-based model are .024 (for iii-V) and .013 (for vii ${ }^{\circ}-\mathrm{ii}$ ). The raised floor for ICP attraction values is due to the assignment of nonzero attraction to all intervals. As Figure 5 shows (by comparison with Figure 2), the net effect is a closer resemblance between the model and the corpus data.[5]

As we have seen, Woolhouse's chord model makes better predictions when the pitch-class model gives attraction values of zero for unisons, thirds, and sixths. Yet the ICP pitch-class model not only gives nonzero values for those intervals, it also makes distinctions among their attraction values that are not based on any direct empirical evidence. It may be that Woolhouse is willing to overlook these problems because the ICP Hypothesis is so simple: all the attraction values come from a single explanatory principle having to do with the lengths of interval cycles. Yet it is far from clear that Occam's razor would lead us to choose ICP over MCP. ICP may be driven by a single, elegant, and numerical principle, but it is hard to imagine how ICP could be implemented cognitively. Do listeners tacitly project intervals into interval cycles and calculate their lengths? Woolhouse does not cite any experimental evidence of this kind of projection. Nor does he explain why this particular faculty would be recruited for processes related to tonal attraction. His closest approach to an explanation of this type is a speculation about how the relationship between interval cycles and tonal attraction might be implemented (cross-domain mapping), but the question of why this relationship might arise is not raised. 


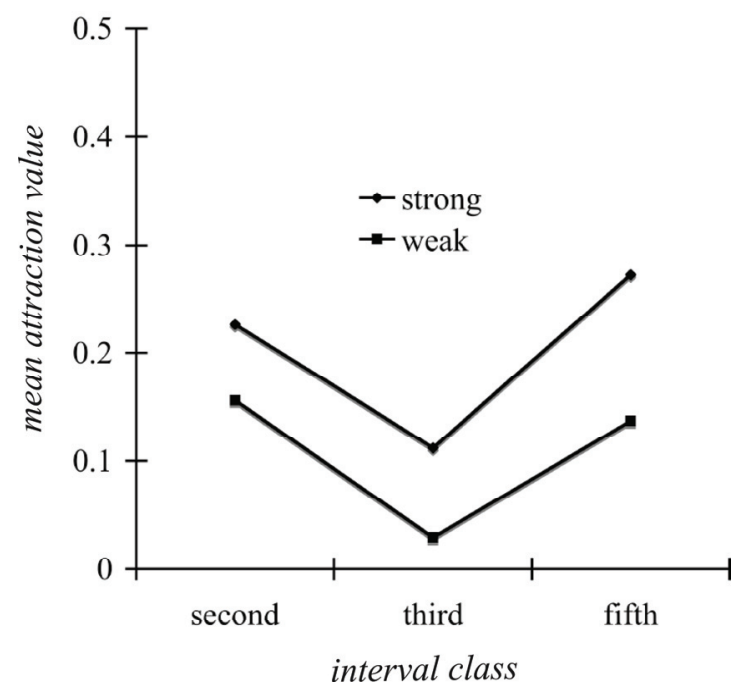

Figure 5. Average attraction value for each progression type according to the MHP-based chord model; compare Figures 1 and 2.

\section{CONCLUSIONS}

Woolhouse starts with the hypothesis that there is a meaningful relationship between the tonal attraction inherent in an interval and the number of times that interval needs to be compounded on itself to yield a whole number of octaves. The foregoing analysis suggests that any such relationship is based on an incidental correlation between an interval's ICP and its MHP. The ICP-based model performs well on the Piston's Table of Usual Root Progressions, but the MHP-based model outperforms the ICPbased model on the corpus data, which is arguably a more important gold standard than Piston's table.

In the final section of his paper, Woolhouse sketches a hypothesis about the emergence of common-practice tonality from the modal system that preceded it. He is interested in the idea that the diatonic scale under the modal system admitted finals on six of the seven scale degrees, but that eventually four of those possibilities fell away, and only the major and minor tonics remained (C and A in the white-note diatonic scale). His hypothesis is that in modal system's heyday, "ICP played little or no part in the cognition of tonal attraction," but that "in the seventeenth century, ICP became increasingly important cognitively." The only evidence he adduces is anecdotal, taking the form of demonstrations that the ICP Hypothesis predicts that the notes of the diatonic scale are collectively attracted to notes of the $\mathrm{C}$ major and A minor triads. I have elsewhere addressed the problems with this line of reasoning (Quinn, 2010). Here I will point out only that the MHP Hypothesis also makes this prediction, and that Woolhouse has a long way to go before establishing that ICP in particular has anything to do with tonal cognition.

My own feeling is that the ICP Hypothesis is not in fact correct, but that Woolhouse's model is otherwise reasonable. Figure 2 and the associated discussion demonstrated why Woolhouse's chord model, when equipped with a pitch-class model that ranks steps and fifths above unisons and thirds in terms of tonal attraction, is capable of distinguishing between strong and weak progressions. This distinction, in turn, helps to explain both Piston's intuitions about chord progressions and Tymoczko's empirical findings.

\section{REFERENCES}

Bharucha, J.J. \& Olney, K.L. (1989). Tonal cognition, artificial intelligence and neural nets. Contemporary Music Review, Vol. 4, No. 1, pp. 341-356.

Budge, H. (1943). A Study of Chord Frequencies Based on the Music of Representative Composers of the Eighteenth and Nineteenth Centuries. New York: Teachers College. 
McHose, A.I. (1937). The Contrapuntal Harmonic Technique of the 18th Century. New York: Appleton-Century-Crofts.

Meeùs, N. (2000). Toward a post-Schoenbergian grammar of tonal and pre-tonal harmonic progressions. Music Theory Online, Vol. 6, No. 1.

Meyer, L.B. (1956). Emotion and Meaning in Music. Chicago: University of Chicago Press.

Ockelford, A. (2006). Implication and expectation in music: A zygonic model. Psychology of Music, Vol. 34, No. 1, pp. 81-142.

Piston, W. (1979). Harmony, 4th ed. New York: Norton.

Piston, W., and DeVoto, M. (1987). Harmony, 5th ed. New York: Norton.

Piston, W. and Westergaard, P. (1968). "Conversation with Walter Piston.” Perspectives of New Music, Vol. 7, No. 1, pp. 3-17.

Povel, D.-J. (2002). A model for the perception of tonal melodies. In: C. Anagnostopoulou, M. Ferrand, \& A. Smaill (Eds.), Music and Artificial Intelligence. Berlin: Springer, pp. 157-166.

Quinn, I. (2010). On Woolhouse's Interval-Class Proximity Hypothesis. Music Theory Spectrum, Vol. 32, No. 2, forthcoming.

Schoenberg, A. (1954). Structural Functions of Harmony. New York: Norton.

Schmuckler, M. (1989). Expectation in music: Investigation of melodic and harmonic processes. Music Perception, Vol. 7, No. 2, pp. 109-150.

Tymoczko, D. (2003). Progressions fondamentales, functions, degrés: Une grammaire de l'harmonie tonale élémentaire. Musurgia, Vol. 10, No. 3-4, pp. 35-64.

Tymoczko, D. (forthcoming). A Geometry of Music. New York: Oxford University Press.

Woolhouse, M. H. (2007). Interval cycles and the cognition of pitch attraction in Western tonalharmonic music. Unpublished doctoral thesis, University of Cambridge, UK.

Woolhouse, M. H. (2009). Modelling tonal attraction between adjacent musical elements. Journal of New Music Research, Vol. 38, No. 4, pp. 357-379.

Woolhouse, M. H. \& Cross, I. (2010). Using interval cycles to model Krumhansl's tonal hierarchies. Music Theory Spectrum. Vol. 32, No. 1, pp. 62-78.

\section{NOTES}

[1] Here, for example, is Piston in an interview with Peter Westergaard: "They [the young] all seem to go through a certain natural evolution. First they are all burned up, the way we were back in the twenties: they want to destroy the past. Then they grow up and say, "Perhaps the past doesn't have to be destroyed after all. I guess I won't destroy it." Then they begin to realize they don't know what the past is, never having studied it. And finally, they get a little older and they look inside themselves to see what they have to say, and they find they have great need for a deep knowledge of their musical heritage. It goes without saying that I except the geniuses." (Piston and Westergaard, 1968, p. 8)

[2] The interval from $p$ to $q$ is $q-p=i$. The interval from $q$ to $p$ is $p-q=-i$.

[3] The basic assumption of a first-order Markov model is that the probability of something occurring at a given position in a sequence depends only on the identity of the thing that occurs in the preceding position. 
[4] Part of the reason the model understates the effect of directionality may be that with Woolhouse's parameter settings of $\beta=4$ and $\gamma=8$ the constant $k$ in equations (3) and (5) is quite small $(1 / 180)$.

[5] These results still show an understated directionality effect, because Woolhouse's parameter settings for $\beta$ and $\gamma$ were left untouched; adjustments to these parameters would yield even better results. 